\title{
Cryptanalysis of Dynamic SHA(2)
}

\author{
Jean-Philippe Aumasson ${ }^{1, \star}$, Orr Dunkelman ${ }^{2, \star \star}$, Sebastiaan Indesteege $\mathrm{e}^{3,4, \star \star \star}$, \\ and Bart Preneel ${ }^{3,4}$ \\ 1 FHNW, Windisch, Switzerland \\ 2 École Normale Supérieure, INRIA, CNRS, Paris, France \\ 3 Department of Electrical Engineering ESAT/COSIC, \\ Katholieke Universiteit Leuven, Belgium \\ ${ }^{4}$ Interdisciplinary Institute for BroadBand Technology (IBBT), Belgium
}

\begin{abstract}
In this paper, we analyze the hash functions Dynamic SHA and Dynamic SHA2, which have been selected as first round candidates in the NIST hash function competition. These hash functions rely heavily on data-dependent rotations, similar to certain block ciphers, e.g., RC5. Our analysis suggests that in the case of hash functions, where the attacker has more control over the rotations, this approach is less favorable than in block ciphers. We present practical, or close to practical, collision attacks on both Dynamic SHA and Dynamic SHA2. Moreover, we present a preimage attack on Dynamic SHA that is faster than exhaustive search.
\end{abstract}

Keywords: Dynamic SHA, Dynamic SHA2, SHA-3 candidate, hash function, collision attack.

\section{Introduction}

New generic cryptanalytic techniques for hash functions [1,2 and the recent results on MD5 and SHA-1 [3, 4,5, along with the fact that the SHA-2 family of hash functions was designed with a similar structure, have led to the initiation of the NIST hash function competition [6], a public competition to develop a new hash standard, which will be called SHA-3.

The competition has sparked a great deal of submissions: 64 new hash function proposals were submitted to the competition, of which 51 were accepted as meeting the submission criteria for the first round. Among the 51 candidates, Dynamic SHA and Dynamic SHA2 stand out as a combination of the SHA family design with data-dependent rotations.

The concept of data-dependent rotations has been explored for block ciphers in several constructions, most notably in the RC5 and RC6 block ciphers [7,8. The security of such block ciphers has been challenged many times, and a majority of attacks is based on guessing the distances of the rotations. In cryptanalysis of

\footnotetext{
* Supported by the Swiss National Science Foundation, project no. 113329.

** This author was supported by the France Telecom chaire.

${ }^{\star \star \star}$ F.W.O. Research Assistant, Fund for Scientific Research — Flanders (Belgium).
} 
hash functions, however, the internal state is known. The attacker even has control over (parts of) the internal state, including rotations, though sometimes this control is only indirect. For example, Mendel et al. 9] exploited data-dependent rotations to find collisions for the hash function of Shin et al. [10. Our attacks on Dynamic SHA and Dynamic SHA2 also exploit data-dependent rotations, to find (second) preimages and collisions.

\section{Brief Description of Dynamic SHA and Dynamic SHA2}

Dynamic SHA and Dynamic SHA2 use similar building blocks, but have different compression functions. This section gives a brief description of these algorithms.

Dynamic SHA and Dynamic SHA2 follow a classical Merkle-Damgård construction, based on a compression function that maps an 8-word chaining value and a 16 -word message to a new 8 -word chaining value. The 256 -bit versions use 32-bit words, and the 512-bit versions use 64-bit words. We focus on the 256-bit versions, also called Dynamic SHA-256 and Dynamic SHA2-256. See [11,12 for details on the 512-bit versions, Dynamic SHA-512 and Dynamic SHA2-512. The following presents a bottom-up description of the compression function, thus starting with its building blocks.

The symbol $\oplus$ stands for exclusive OR $(\mathrm{XOR}), \wedge$ for logical AND, $\vee$ for logical $\mathrm{OR}$, and + for integer addition. Numbers in hexadecimal basis are written in typewriter font (e.g., FF = 255). We count bit indices starting from zero at the least significant bit (LSB). Thus, the first bit of a word $w$ is written as $w^{0}$, and more generally we use the notation $w^{i}$ for the bit $i$ of the word $w$. The most significant bit (MSB) of $w$ is thus $w^{31}$ for Dynamic SHA-256, and $w^{63}$ for Dynamic SHA-512. Note that the $i$-th bit of a word corresponds to the bit number $i-1$, since we start counting from zero.

\subsection{Building Blocks}

The function $G$ takes as input three words $x_{1}, x_{2}, x_{3}$ and an integer $t \in\{0,1,2,3\}$, and returns one word, computed as follows:

$$
G_{t}\left(x_{1}, x_{2}, x_{3}\right)=\left\{\begin{array}{ll}
x_{1} \oplus x_{2} \oplus x_{3} & \text { if } t=0 \\
\left(x_{1} \wedge x_{2}\right) \oplus x_{3} & \text { if } t=1 \\
\left(x_{1} \wedge x_{2}\right) \oplus x_{3} \oplus \neg x_{1} & \text { if } t=2 \\
\left(x_{1} \wedge x_{2}\right) \oplus x_{3} \oplus \neg x_{2} & \text { if } t=3
\end{array} .\right.
$$

Note that this definition is simplified, but equivalent to the original in [1, 12.

The function $R$ takes as input eight words $x_{1}, \ldots, x_{8}$ and an integer $t$, and returns one word computed as follows:

$$
\left.R\left(x_{1}, \ldots, x_{8}, t\right)=\left(\left(\left(\left(\left(\left(x_{1} \oplus x_{2}\right)+x_{3}\right) \oplus x_{4}\right)+x_{5}\right) \oplus x_{6}\right)+x_{7}\right) \oplus x_{8}\right) \ggg t .
$$

The function $R 1$ takes as input eight words $x_{1}, \ldots, x_{8}$ and returns one word computed as follows (in the 256-bit versions): 


$$
\begin{aligned}
& \left.t_{0} \leftarrow\left(\left(\left(\left(x_{1}+x_{2}\right) \oplus x_{3}\right)+x_{4}\right) \oplus x_{5}\right)+x_{6}\right) \oplus x_{7} \\
& t_{1} \leftarrow\left(\left(t_{0} \gg 17\right) \oplus t_{0}\right) \wedge 0001 \mathrm{FFFF} \\
& t_{2} \leftarrow\left(\left(t_{1} \gg 10\right) \oplus t_{1}\right) \wedge 000003 \mathrm{FF} \\
& t_{3} \leftarrow\left(\left(t_{2} \gg 5\right) \oplus t_{2}\right) \wedge 0000001 \mathrm{~F} \\
& \text { return } x_{8} \ggg t_{3}
\end{aligned}
$$

Finally, the COMP function takes as input eight words $a, \ldots, h$ representing the internal state, eight message words $w_{0}, \ldots, w_{7}$, or $w_{8}, \ldots, w_{15}$, and an integer $t$. COMP updates the internal state as follows (in the 256-bit versions):

$$
\begin{array}{l|l}
T \leftarrow R\left(a, \ldots, h, w_{t} \bmod 32\right) & T \leftarrow R\left(a, \ldots, h,\left(w_{t} \gg 15\right) \bmod 32\right) \\
h \leftarrow g & h \leftarrow g+w_{t+7} \\
g \leftarrow f \gg\left(\left(w_{t} \gg 5\right) \bmod 32\right) & g \leftarrow f \gg\left(\left(w_{t} \gg 20\right) \bmod 32\right) \\
f \leftarrow e+w_{t+3} & f \leftarrow e+w_{t+6} \\
e \leftarrow d \gg\left(\left(w_{t} \gg 10\right) \bmod 32\right) & e \leftarrow d \gg\left(\left(w_{t} \gg 25\right) \bmod 32\right) \\
d \leftarrow G_{w_{t} \gg 30}(a, b, c)+w_{t+2} & d \leftarrow G_{t \bmod 4}(a, b, c)+w_{t+5} \\
c \leftarrow b & c \leftarrow b+w_{t} \\
b \leftarrow a & b \leftarrow a \\
a \leftarrow T+w_{t+1} & a \leftarrow T+w_{t+4}
\end{array}
$$

\subsection{Compression Functions}

Given a chaining value $h_{0}, \ldots, h_{7}$ and a message block $w_{0}, \ldots, w_{15}$, the compression function of Dynamic SHA (Dynamic SHA2, respectively) produces a new chaining value, as described in Algorithm 1 (Algorithm 2, resp.).

The compression function of Dynamic SHA is composed of an initialization, an iterative part of 48 rounds, and a feedforward of the initial chaining value. It uses three constants $T T_{0}, T T_{1}, T T_{2}$.

The compression function of Dynamic SHA2 is composed of an initialization followed by three iterative parts, and finally by a feedforward. Note that, when calling COMP with the message words $w_{8}, \ldots, w_{15}$ and an integer $t, w_{t}$ stands for $w_{8}, w_{t+1}$ stands for $w_{9}$, etc. Dynamic SHA2 surprisingly enough, uses no constants.

\section{Collision Attack on Dynamic SHA}

This section describes a practical collision attack on Dynamic SHA. It builds on a 9-step local collision that exploits an important differential property of the function $R 1$, which we introduce first. The same local collision pattern is repeated three times to find collisions for the entire compression function. Furthermore, these three instances of the local collision pattern can be decoupled, which drastically reduces the attack complexity. We present the attack on Dynamic SHA-256 here. We could adapt it to Dynamic SHA-512 with only minimal changes, as detailed in Appendix C. 


\section{Algorithm 1. Compression function of Dynamic SHA}

Initialization

$$
a=h_{0} \quad b=h_{1} \quad c=h_{2} \quad d=h_{3} \quad e=h_{4} \quad f=h_{5} \quad g=h_{6} \quad h=h_{7}
$$

Iterative part

$$
\begin{aligned}
& \text { for } t=0,1 \ldots, \\
& \qquad \begin{aligned}
& 4 \\
& \leftarrow R 1(a, b, c, d, e, f, g, h) \\
& U \leftarrow G(a, b, c, t \bmod 4)+w_{t \bmod 16}+T T_{t \gg 4} \\
& (a, b, c, d, e, f, g, h) \leftarrow(T, a, b, U, d, e, f, g)
\end{aligned}
\end{aligned}
$$

Feedforward

$$
\begin{array}{llll}
h_{0} \leftarrow h_{0}+a & h_{1} \leftarrow h_{1}+b & h_{2} \leftarrow h_{2}+c & h_{3} \leftarrow h_{3}+d \\
h_{4} \leftarrow h_{4}+e & h_{5} \leftarrow h_{5}+f & h_{6} \leftarrow h_{6}+g & h_{7} \leftarrow h_{7}+h
\end{array}
$$

\section{Algorithm 2. Compression function of Dynamic SHA2}

Initialization

$$
a=h_{0} \quad b=h_{1} \quad c=h_{2} \quad d=h_{3} \quad e=h_{4} \quad f=h_{5} \quad g=h_{6} \quad h=h_{7}
$$

First iterative part

$$
\begin{aligned}
& \operatorname{COMP}\left(a, b, c, d, e, f, g, h, w_{0}, w_{1}, \ldots, w_{7}, 0\right) \\
& \operatorname{COMP}\left(a, b, c, d, e, f, g, h, w_{8}, w_{9}, \ldots, w_{15}, 0\right)
\end{aligned}
$$

Second iterative part

$$
\begin{aligned}
& \text { for } t=0,1 \ldots, 8 \\
& \qquad \begin{aligned}
T \leftarrow R 1(a, b, c, d, e, f, g, h) \\
(a, b, c, d, e, f, g, h) \leftarrow(T, a, b, c, d, e, f, g)
\end{aligned}
\end{aligned}
$$

Third iterative part

$$
\begin{aligned}
& \text { for } t=1,2 \ldots, 7 \\
& \operatorname{COMP}\left(a, b, c, d, e, f, g, h, w_{0}, w_{1}, \ldots, w_{7}, t\right) \\
& \operatorname{COMP}\left(a, b, c, d, e, f, g, h, w_{8}, w_{9}, \ldots, w_{15}, t\right)
\end{aligned}
$$

Feedforward

$$
\begin{array}{llll}
h_{0} \leftarrow h_{0}+a & h_{1} \leftarrow h_{1}+b & h_{2} \leftarrow h_{2}+c & h_{3} \leftarrow h_{3}+d \\
h_{4} \leftarrow h_{4}+e & h_{5} \leftarrow h_{5}+f & h_{6} \leftarrow h_{6}+g & h_{7} \leftarrow h_{7}+h
\end{array}
$$




\subsection{A Differential Property of the Function $R 1$}

To overcome the obstacle of data-dependent rotation, our attack ensures that no difference occurs in any of the data-dependent rotation amounts. This section clarifies how to achieve this.

The data-dependent rotations are located in the 8-input function $R 1$. For Dynamic SHA-256, consider the difference $\Delta=80004000$, i.e., only bits 31 and 14 are set. Let one of the first seven inputs to the function $R 1$ have this difference, i.e., one of $x_{1}, \ldots, x_{7}$. In the first step of $R 1$, an intermediary word $t_{0}$ is computed as follows:

$$
t_{0} \leftarrow\left(\left(\left(\left(\left(x_{1}+x_{2}\right) \oplus x_{3}\right)+x_{4}\right) \oplus x_{5}\right)+x_{6}\right) \oplus x_{7} .
$$

The difference in the MSB always propagates to $t_{0}$. Assuming that no carry occurs for bit 14, the intermediary $t_{0}$ also has the difference $\Delta$. If $t_{0}$ has a difference $\Delta$, this difference is then absorbed by the rest of the function $R 1$. Indeed, the next step computes the intermediary word $t_{1}$ as

$$
t_{1} \leftarrow\left(\left(t_{0} \gg 17\right) \oplus t_{0}\right) \wedge 0001 \mathrm{FFFF} .
$$

Note that $(\Delta \gg 17) \oplus \Delta=80000000$, which is absorbed by the logical AND operation. We note that there are other differences of Hamming weight 2 that exhibit the same property and may be used without any change in the attack, e.g., $\Delta=80000010$.

We now estimate the probability that a single $\Delta$-difference in one of the first seven inputs of the function $R 1$ is absorbed. As a $\Delta$-difference in $t_{0}$ is absorbed with certainty, it suffices that a $\Delta$-difference in one of the seven first inputs propagates to $t_{0}$. This happens when no carry difference occur for bit 14 in any of the modular additions. The probability that a one-bit difference in one of the summands in an addition does not cause a carry difference is $1 / 2$. Thus, the probability that a $\Delta$-difference is absorbed by the function $R 1$ can be estimated to $2^{-k}$, where $k$ is the number of modular additions the difference propagates through. For instance, a difference in $x_{3}$ activates two modular additions, so $k=2$.

However, the actual probability is higher, as the undesirable effects of a carry difference in one modular addition can be reverted by another carry difference in a subsequent addition. The combination of modular additions and XOR can be represented compactly in a trellis, and a variant of the Viterbi algorithm can be used to efficiently count the probability that a $\Delta$-difference is passed to $t_{0}$ unchanged. Our computer aided research revealed that this is indeed an important effect: For a difference in $x_{3}$ or $x_{4}$, the actual probability is $2^{-1.58}$ rather than $2^{-2}$, and for a difference in $x_{1}$ or $x_{2}$, the actual probability is $2^{-2.07}$ rather than $2^{-3}$. For differences in the other words, only one modular addition is affected, so no carry differences can be canceled. Hence, in those cases, the simple estimation is correct. 
Table 1. A 9-step local collision for Dynamic SHA. The difference at step $t$ is the difference in the state before computing step $t$.

\begin{tabular}{c|cccccccc|c|c}
\hline$t$ & $a$ & $b$ & $c$ & $d$ & $e$ & $f$ & $g$ & $h$ & $w$ & $\operatorname{Pr}$ \\
\hline 0 & 0 & 0 & 0 & 0 & 0 & 0 & 0 & 0 & $\Delta$ & $2^{-1}$ \\
1 & 0 & 0 & 0 & $\Delta$ & 0 & 0 & 0 & 0 & 0 & $2^{-1.58}$ \\
2 & 0 & 0 & 0 & 0 & $\Delta$ & 0 & 0 & 0 & 0 & $2^{-1}$ \\
3 & 0 & 0 & 0 & 0 & 0 & $\Delta$ & 0 & 0 & 0 & $2^{-1}$ \\
4 & 0 & 0 & 0 & 0 & 0 & 0 & $\Delta$ & 0 & 0 & 1 \\
5 & 0 & 0 & 0 & 0 & 0 & 0 & 0 & $\Delta$ & 0 & $2^{-5}$ \\
6 & $\Delta$ & 0 & 0 & 0 & 0 & 0 & 0 & 0 & 0 & $2^{-2.07} \cdot 2^{-2}$ \\
7 & 0 & $\Delta$ & 0 & 0 & 0 & 0 & 0 & 0 & 0 & $2^{-2.07} \cdot 2^{-2}$ \\
8 & 0 & 0 & $\Delta$ & 0 & 0 & 0 & 0 & 0 & $\Delta$ & $2^{-1.58} \cdot 2^{-1}$ \\
\hline & 0 & 0 & 0 & 0 & 0 & 0 & 0 & 0 & & \\
\hline
\end{tabular}

\subsection{A 9-Step Local Collision}

We present a simple 9-step local collision for Dynamic SHA in Table 1. A difference of $\Delta=80004000$ is introduced, then, all further diffusion of this difference is avoided. After seven more steps, the difference has rotated through the internal state of Dynamic SHA once, and can be canceled via an appropriate difference in the message word. The characteristic has probability $2^{-20.3}$.

In step 0 , a $\Delta$-difference is introduced via the message word. Note that the message word itself can contain any additive difference that can cause a $\Delta$ difference in the state. In steps 1 to 4 , the $\Delta$-difference in one of the state variables is absorbed by the function $R 1$, as described in Section 3.1. Then, at the beginning of step 5 , there is a $\Delta$-difference in the internal state word $h$. This word is rotated by a data-dependent amount, and thus we can require that it is rotated by zero bits, i.e., not rotated at all. In steps 6 and 7 , the $\Delta$-difference should be absorbed by the $G$-functions. Any $G$-function except XOR absorbs differences in its first two inputs with probability $1 / 2$ per bit. Also, $R 1$ should absorb the differences in these steps. Finally, in step 8, the difference in the state variable $c$ is canceled by another $\Delta$-difference coming from the message word.

The probability that the local collision pattern is followed is estimated by simply multiplying the probabilities of all the events discussed above. The probabilities of each step are indicated in Table 1. This yields an overall probability of $2^{-20.3}$ for the entire 9 -step local collision.

\subsection{The Attack}

Our attack repeats the 9 -step collision three times. This made possible by the simple message schedule, which consists of a simple repetition of the 16 words in a message block. Thus, the only message words that have a difference are $w_{0}$, which introduces the differences, and $w_{8}$, which cancels them.

A straightforward attack would consist of choosing an arbitrary message block, and applying a difference of $\Delta=80004000$ to $w_{0}$ and $w_{8}$. As the local collision 
is repeated three times, the complexity of this attack would be approximately $\left(2^{20.3}\right)^{3}=2^{61}$. This can be improved tremendously by making the three local collisions independent. Then, the three local collision complexities can be added rather than multiplied.

The first two local collisions can be decoupled in a straightforward manner as only the message words $w_{0}$ to $w_{8}$ influence the first local collision. Therefore, once suitable values for these message words have been found, there is still enough freedom remaining in the other message words. The words $w_{0}$ to $w_{8}$ can thus be kept constant, while values for $w_{9}$ to $w_{15}$ are searched such that the second local collision is also achieved.

Controlling Internal State Values. In each step of Dynamic SHA, the new value of the internal state word $d$ is found as the modular addition of a message word and an intermediate depending on the internal state words $a, b$ and $c$. Full control over message words allows an adversary to give the internal state word $d$ any desired value. Indeed, it holds that

$$
w_{t \bmod 16}=d_{\text {new }}-G(a, b, c, t \bmod 4)-T T_{t \gg 4} .
$$

Applying this to eight consecutive steps allows one to almost fully control the final internal state. In every step, the new value of $d$ is fixed to some desired value. These values then shift through the internal state words a number of times, to end up as one of the internal state words after the eighth step. However, a complication arises with the first three steps, which ends up in the state words $a$, $b$ and $c$. Before a controlled value from $d$ ends up in one of these three state words, it is be rotated by a data-dependent amount. An obvious way to sidestep this issue is to choose a rotation-invariant value for these three words, i.e., 00000000 or FFFFFFFF. Then, the data-dependent rotations have no influence.

Decoupling All Three Local Collisions. Our attack consists of three phases, each dealing with one local collision. The first phase satisfies the first local collision, using the message words $w_{0}$ to $w_{8}$. It would be possible to use message modification techniques here to find a conforming message pair quicker, but as the later phases of the attack dominate the overall complexity anyway, no significant gains can be made in this way.

To satisfy the second local collision, we use the freedom in the remaining message words. However, we do not choose the remaining message words directly, but rather choose the internal state after step 15. We then use the words $w_{8}$ to $w_{15}$ to connect to this state, using the technique outlined earlier. We fix the values of $a, b$ and $c$ to zero, to make them rotation-invariant, and choose the remaining five words arbitrarily. Note that $w_{8}$ was already determined in phase 1 , so it should not be modified again, but $w_{8}$ is used here to force a zero value, which ends up in the internal state word $d$ after step 15 . This issue is solved by shifting this condition on $w_{8}$ to phase 1 . Instead of arbitrarily choosing $w_{8}$ there, it is computed such that the required zero is generated. This does not change the complexity of the first phase. 
Finally, to satisfy the third local collision, we modify $w_{7}$. Then, only $d$ changes after step seven. As the value in $w_{8}$, which should force $d$ to zero after step eight, depends only on the internal state words $a, b$ and $c$ before step eight, modifying $w_{7}$ does not require a correction in $w_{8}$. Thus, such modifications do not change the fact that the first local collision pattern is followed. The values of $w_{9}$ to $w_{15}$ are then updated such that the internal state after step 15 is unchanged, and so the start of the second local collision will be unaltered. For the same reasons as before, the change in $w_{7}$ also does not affect the end of the second local collision pattern.

Hence, we dispose of a modification algorithm that leaves the first two local collisions unaffected, but changes the internal state values before the third local collision randomly. This provides the required freedom to also satisfy this third and final local collision. Hence, the overall attack complexity can be estimated at about $2^{21}$ Dynamic SHA compression function computations. Appendix A reports on our implementation of the attack, with an example of collision.

\section{Preimage Attack on Dynamic SHA}

This section describes (first and second) preimage attacks on Dynamic SHA. We first describe how to find preimages for the compression function of Dynamic SHA, and then explain how to extend this to first and second preimage attacks. on the Dynamic SHA hash function. We describe how to attack Dynamic SHA256 here, and refer to Appendix C for details on how to adapt the attack to Dynamic SHA-512.

Conceptually, our preimage attack bears some similarity to the work on SHA-0 and SHA-1 by De Cannière and Rechberger [13, for it finds a preimage bit slice per bit slice. If all data-dependent rotation amounts in Dynamic SHA are assumed to be zero, then a bit of any intermediate word cannot be influenced by any other bit of higher position. This is because, besides rotations, all operations are either bit-wise or modular additions.

\subsection{Preimage Attack on the Compression Function}

Assume that the rotations in a block of Dynamic SHA are all zero. Then, all words in Dynamic SHA can be divided into bit slices, as all computations are now T-functions [14. As noted above, bit $i$ of each word can only be influenced by bits 0 to $i$ of other words. When bits 0 to $(i-1)$ of each word are known, bit $i$ of all words can be determined.

In a preimage attack on the Dynamic SHA compression function, the internal state is given before step 0 and after step 47. Our attack starts by determining the LSB of each word. To determine this bit of all of the internal state words in every step, only the LSBs of the 16 message words need to be known. There are $2^{16}$ choices for these 16 bits. Then, it can be verified whether the LSBs of the eight internal state words after step 47 are correct. This occurs with probability $2^{-8}$, so $2^{8}$ choices are expected to survive. 
We then proceed to the next bit slice. Keeping the choice for the LSB slice fixed, the same procedure can be repeated. For each choice of the LSB slice again $2^{8}$ choices for the second LSB are expected to survive. For Dynamic SHA-256, this procedure is repeated until the $28 \mathrm{LSBs}$ (bits 0-27) have been determined. At that point, one of the bits of each of the 48 rotation constants can be determined, as it does not depend on the higher bits of any word. Now, it can be verified if the initial assumption that all rotation constants are zero indeed holds. This corresponds to a 48-bit condition, i.e., for all rotation constants to be zero, surely this single bit of each rotation constant has to be zero. Any choices that do not satisfy this condition are eliminated. Then, the next bit is determined as before, after which another bit of each rotation constant can be verified. This is repeated until all bits have been determined.

\subsection{Complexity Evaluation}

The attack can be described as a simple tree search, where a tree level corresponds to a bit slice, and a node represents an assignment for all bits in the slice under consideration, and all LSB slices. To expand a node in the tree, one guesses the 16 message bits of the next slice, and checks that the conditions on the state words after step 47 are satisfied. As explained above, on average about $2^{8}$ choices are expected to survive, i.e., the tree has a branching factor of $2^{8}$. When the 28 LSB slices are known, however, the average number of child nodes drops by $2^{-48}$ due to the additional filtering. The cost of expanding one node is about $2^{16}$ Dynamic SHA compression function evaluations, as $2^{16}$ choices have to be investigated. The expected number of solutions is equal to the expected number of nodes at the deepest level of the tree, which is $2^{8 \cdot 32} \cdot 2^{-48 \cdot 5}=2^{16}$. This agrees with the observation that for a given input/output chaining values of the compression function, there are expected to be $2^{256}$ message blocks that conform to this combination. For each of these, the probability that all the rotations are by 0 positions is $2^{-240}$, so about $2^{16}$ remain.

As we aim to find just one solution, i.e., any node on the deepest level of the tree, a depth-first search is well suited to our application. It requires only negligible memory and can easily be parallelised. Since, for Dynamic SHA-256, $2^{16}$ solutions are expected, the depth-first search needs to search only about a fraction $2^{-16}$ of the entire tree before encountering the first solution. Due to the large branching factor, the total number of nodes in the tree is well approximated by the number of nodes on the widest level of the tree, which has $2^{8 \cdot 27}=2^{216}$ nodes for Dynamic SHA-256. The search is thus expected to expand about $2^{200}$ nodes, each of which costs $2^{16}$ Dynamic SHA-256 compression function evaluations, resulting in a total attack complexity of $2^{216}$ Dynamic SHA256 compression function evaluations.

\subsection{Application to the Hash Function}

Our preimage attack on the compression function directly gives a second preimage attack on the Dynamic SHA hash function with the same complexity, 
provided that there is at least one message block that does not contain any padding in the challenge message.

For a first preimage, the padding bits limit the control an attacker has over the message bits. It is not possible to simply copy the padding as in a second preimage attack. Thus, we use the following approach instead. First, choose a message length such that the last padded message block only contains 65 bits of padding, which is the minimum. Then, choose an arbitrary message for all but the last message block. Finally, a modified version of the attack in Section 4.1] is used to determine the last message block.

The main difference is that the last 65 bits of the message block can not be chosen by the adversary, as they are padding bits. Their contents are fixed by the choice of the message length. However, the same approach as in Section 4.1 can still be applied, except that fewer bits can be chosen in each bit slice. For Dynamic SHA-256, the expected number of solutions in the search tree now becomes $2^{6 \cdot 27} \cdot 2^{-42 \cdot 4} \cdot 2^{-43 \cdot 1}=2^{-49}$. A solution is thus only expected to exist with probability $2^{-49}$, thus the attack is repeated sufficiently many times with a different message length. The number of nodes at the widest level of the tree is $2^{6 \cdot 27}$, and the cost for expanding a single node at this level is $2^{14}$ Dynamic SHA compression function calls. Thus, the total attack complexity becomes approximately $2^{49} \cdot 2^{6 \cdot 27} \cdot 2^{14}=2^{225}$ Dynamic SHA compression function evaluations.

\section{Collision Attack on Dynamic SHA2}

To attack Dynamic SHA2, we use similar ideas as for Dynamic SHA. Specifically, we use the control of the message to ensure that as many rotations as possible are by the amounts that we need. Moreover, as many of the rotations amounts are directly determined by the message, our task becomes easier. Our attack is based on introducing a difference in the most significant bit of two message words, $w_{8}$ and $w_{14}$. As a 32 -bit condition is imposed on the chaining value, a twoblock collision finding technique is used, where the first block is searched until a suitable chaining value is encountered. We describe our attack on Dynamic SHA2-256 here. It can be adapted to Dynamic SHA2-512, as Appendix C shows.

\subsection{First Iterative Part}

Given an initial value $a, \ldots, h$, the first iterative part of the compression function of Dynamic SHA2 updates the chaining value words $a, \ldots, h$ by computing

$$
\operatorname{COMP}\left(a, b, \ldots, h, w_{0}, w_{1}, \ldots, w_{7}\right),
$$

Since there is no difference in the message words $w_{0}, \ldots, w_{7}$ nor in the initial value, we have no difference at this stage.

Then, Dynamic SHA2 computes

$$
\operatorname{CoMP}\left(a, b, \ldots, h, w_{8}, w_{9}, \ldots, w_{15}\right) .
$$


To follow our characteristic, the difference in $w_{8}$ and in $w_{14}$ should lead to a difference $\Delta=80000000$ in $c$ and in $f$. Below, we show that, to obtain these differences, it suffices to set $w_{8}^{30}=1$ and to ensure that $b$ equals FFFFFFF after the first COMP. These conditions are easily satisfied, and do not increase the complexity of our attack.

We note that $w_{14}$ is used only once in the first iterative part. Thus the difference $\Delta$ in $w_{14}$ only propagates to $f$, when COMP sets $f \leftarrow e+w_{14}$. The word $w_{8}$, however, is used eight times, but as only the MSB has a difference, only two of these require our attention: first, when setting $c \leftarrow b+w_{8}$ (which gives the difference $\Delta$ in $c$ with probability one), and second when setting

$$
d \leftarrow G_{w_{8} \gg 30}(a, b, c)+w_{10} .
$$

Here, the two MSBs of $w_{8}$ encode the index of the function used in $G$. Since we have a difference in the MSB of $w_{8}$, different functions are applied to $(a, b, c)$. To obtain the same output, we require that the functions $G_{1}$ and $G_{3}$ are used, that is, we set the bit $w_{8}^{30}=1$. The reason for this is that, when $b$ equals FFFFFFF, it is ensured that the outputs of both functions are equal, as can readily be seen from the definition of the $G$-functions in Section 2.1

To summarize, a difference $\Delta$ in $w_{8}$ and $w_{14}$ yields a difference $\Delta$ in $c$ and $f$ after the first iterative part. To have $b=$ FFFFFFFF, it is sufficient to start from a chaining values that gives at the very first COMP a $T$ such that $T+w_{1}=$ FFFFFFFF. Such a chaining value can be reached in about $2^{32}$ trials, and needs to be precomputed only once. That is, one first needs to find a message block leading to a chaining value that satisfies $T+w_{1}=$ FFFFFFFF, before starting the actual differential attack with a second block. Actually, by using the freedom in $w_{0}$ and $w_{1}$ rather than fixing them a priori, this step can be accelerated further. However, as the other parts of the attack dominate the overall complexity, no significant gains can be made in this way.

\subsection{Second Iterative Part}

Table 2 describes our differential characteristic for the second iterative part of Dynamic SHA2. Note that no message word enters this part. A set of conditions that ensure that this characteristic is followed, is relatively simple. Indeed, except when $t=2$ and $t=5$, the two differences $\Delta$ vanish in the first step of the computation of $R 1$, namely when computing

$$
((((a+b) \oplus c)+d) \oplus e)+f) \oplus g .
$$

Therefore, particular conditions are only required for $t=2$ and $t=5$.

When $t=2$, the difference in $e$ gives a difference of 16 in the rotation amounts, and so the function $R 1$ returns $h \ggg r$ and $(h \oplus \Delta) \ggg(r+16 \bmod 32)$, respectively. In order to obtain, as required by our differential characteristic, the relation

$$
(h \ggg r) \oplus \Delta=(h \oplus \Delta) \ggg(r+16 \bmod 32),
$$


Table 2. Differential characteristic for the second iterative part of Dynamic SHA2. The difference at step $t$ is the difference in the state before computing step $t$.

\begin{tabular}{c|cccccccc}
\hline$t$ & $a$ & $b$ & $c$ & $d$ & $e$ & $f$ & $g$ & $h$ \\
\hline 0 & 0 & 0 & $\Delta$ & 0 & 0 & $\Delta$ & 0 & 0 \\
1 & 0 & 0 & 0 & $\Delta$ & 0 & 0 & $\Delta$ & 0 \\
2 & 0 & 0 & 0 & 0 & $\Delta$ & 0 & 0 & $\Delta$ \\
3 & $\Delta$ & 0 & 0 & 0 & 0 & $\Delta$ & 0 & 0 \\
4 & 0 & $\Delta$ & 0 & 0 & 0 & 0 & $\Delta$ & 0 \\
5 & 0 & 0 & $\Delta$ & 0 & 0 & 0 & 0 & $\Delta$ \\
6 & $\Delta$ & 0 & 0 & $\Delta$ & 0 & 0 & 0 & 0 \\
7 & 0 & $\Delta$ & 0 & 0 & $\Delta$ & 0 & 0 & 0 \\
8 & 0 & 0 & $\Delta$ & 0 & 0 & $\Delta$ & 0 & 0 \\
\hline
\end{tabular}

a sufficient condition is to have $r=16$, and $h$ invariant under 16-bit rotation, i.e., $(h \ggg 16)=h$. This means that $h$ should be of the form XYZTXYZT, which we call symmetric. When $t=5$, we require similar conditions.

Now, observe that the words that should be symmetric are $c$ and $f$ obtained after the first iterative part. The values of $c$ and $f$ then directly depend on $w_{8}$ and $w_{14}$ (see description of COMP in Section 2). We now have to find values of $w_{8}$ and of $w_{14}$ that give symmetric $c$ and $f$.

Such $w_{8}$ and $w_{14}$ can be found as follows: first fix $w_{14}$ to some arbitrary value, and search for a $w_{8}$ that gives a symmetric $c$, in $2^{16}$ trials. Then, fix $w_{8}$ to the value found, and search for a pair $\left(w_{5}, w_{14}\right)$ that gives a symmetric $f$ after the first iterative part. Here we need $w_{5}$ to have enough freedom, since for certain choices of $w_{5}$, there does not exist a suitable $w_{14}$. Again, $2^{16}$ trials are expected. Then we are enough degrees of freedom in the message words that do not affect $c$ and $f$ to find rotation $r=16$.

Assuming symmetric $c$ and $f$ after the first iterative part, the characteristic is followed with probability $2^{-10}$, since the condition $r=16$ is satisfied for both $t=2$ and $t=5$ with probability $2^{-5} \times 2^{-5}$. By trying several values of, for example, $w_{9}$, and leaving the other message words fixed, one can thus find a conforming message pair for the first two iterative parts in about $2^{10}$ trials.

\subsection{Third Iterative Part}

Given the final difference of the second iterative part, we found a characteristic for the second round that yields no difference in the final state, thus given a collision. Table 6in Appendix B]describes our differential characteristic. Appendix B also explains in detail why the characteristic can be followed with probability $2^{-42}$, given some conditions on the input.

Combining our differential characteristics with their respective conditions on the message, we obtain a method for finding a 2-block collision in about $2^{42+10}=$ $2^{52}$ trials. The attack succeeds with probability close to one. 
Table 3. Summary of our results

\begin{tabular}{|c|c|c|c|}
\hline Hash Function & Attack & Complexity & Section \\
\hline Dynamic SHA-256 & Collision & $2^{21}$ & 3 \\
\hline Dynamic SHA-512 & Collision & $2^{22}$ & $3 \mathrm{C}$ \\
\hline Dynamic SHA-256 & Second preimage & $2^{216}$ & 4 \\
\hline Dynamic SHA-512 & Second preimage & $2^{256}$ & $4 \overline{4 C}$ \\
\hline Dynamic SHA-256 & First preimage & $2^{225}$ & 4 \\
\hline Dynamic SHA-512 & First preimage & $2^{262}$ & $4 \mathrm{C}$ \\
\hline Dynamic SHA2-256 & Collision & $2^{52}$ & 5 \\
\hline Dynamic SHA2-512 & Collision & $2^{85}$ & 5 \\
\hline
\end{tabular}

\section{Conclusion}

In this paper we have discussed the security of the two SHA-3 candidates Dynamic SHA and Dynamic SHA2. We have analyzed their security, and found out that, despite their reliance on data-dependent rotations and in the case of Dynamic SHA2 even data-dependent functions, their security is subverted by the vast control and knowledge the adversary has while attacking a hash function. We also showed that neither Dynamic SHA nor Dynamic SHA2 are suitable to be selected as SHA-3, following their lack of security. Table 3 summarizes our results.

\section{Acknowledgements}

The research presented in this paper was performed in part while the authors were visiting Schloss Dagstuhl (http://www.dagstuhl.de/) in January 2009.

This work was supported in part by the IAP Programme P6/26 BCRYPT of the Belgian State (Belgian Science Policy), and in part by the European Commission through the ICT programme under contract ICT-2007-216676 ECRYPT II.

\section{References}

1. Kelsey, J., Schneier, B.: Second preimages on n-bit hash functions for much less than $2^{n}$ work. In: [15], pp. 474-490

2. Kelsey, J., Kohno, T.: Herding hash functions and the Nostradamus attack. In: Vaudenay, S. (ed.) EUROCRYPT 2006. LNCS, vol. 4004, pp. 183-200. Springer, Heidelberg (2006)

3. Wang, X., Yu, H.: How to break MD5 and other hash functions. In: [15], pp. 19-35

4. De Cannière, C., Rechberger, C.: Finding SHA-1 characteristics: General results and applications. In: Lai, X., Chen, K. (eds.) ASIACRYPT 2006. LNCS, vol. 4284, pp. 1-20. Springer, Heidelberg (2006)

5. Stevens, M., Lenstra, A.K., de Weger, B.: Chosen-prefix collisions for MD5 and colliding X.509 certificates for different identities. In: Naor, M. (ed.) EUROCRYPT 2007. LNCS, vol. 4515, pp. 1-22. Springer, Heidelberg (2007) 
6. National Institute of Standards and Technology. Cryptographic hash algorithm competition, http://www.nist.gov/hash-competition

7. Rivest, R.L.: The RC5 encryption algorithm. In: Preneel, B. (ed.) FSE 1994. LNCS, vol. 1008, pp. 86-96. Springer, Heidelberg (1995)

8. Rivest, R.L., Robshaw, M.J.B., Yin, Y.L.: RC6 as the AES. In: AES Candidate Conference, pp. 337-342 (2000)

9. Mendel, F., Pramstaller, N., Rechberger, C.: Improved collision attack on the hash function proposed at PKC 1998. In: Rhee, M.S., Lee, B. (eds.) ICISC 2006. LNCS, vol. 4296, pp. 8-21. Springer, Heidelberg (2006)

10. Shin, S.U., Rhee, K.H., Ryu, D., Lee, S.: A new hash function based on MDxfamily and its application to MAC. In: Imai, H., Zheng, Y. (eds.) PKC 1998. LNCS, vol. 1431, pp. 234-246. Springer, Heidelberg (1998)

11. Xu, Z.: Dynamic SHA. Submission to NIST (2008)

12. Xu, Z.: Dynamic SHA2. Submission to NIST (2008)

13. De Cannière, C., Rechberger, C.: Preimages for reduced SHA-0 and SHA-1. In: Wagner, D. (ed.) CRYPTO 2008. LNCS, vol. 5157, pp. 179-202. Springer, Heidelberg (2008)

14. Klimov, A., Shamir, A.: Cryptographic applications of t-functions. In: Matsui, M., Zuccherato, R.J. (eds.) SAC 2003. LNCS, vol. 3006, pp. 248-261. Springer, Heidelberg (2004)

15. Cramer, R. (ed.): EUROCRYPT 2005. LNCS, vol. 3494. Springer, Heidelberg (2005)

\section{A Practical Results}

We have implemented our collision attack on Dynamic SHA. Collisions for Dynamic SHA-256 and Dynamic SHA-512 are found in a matter of seconds on an average desktop PC. A collision example for Dynamic SHA-256 is given in Table 4. An all-zero block was appended to both messages to circumvent an error in the padding routine of the Dynamic SHA reference implementation, which causes part of the last message block to be reused in the padding block.

Table 4. Collision example for Dynamic SHA-256: two messages and their common digest

\begin{tabular}{|c|c|c|c|c|c|c|c|}
\hline 34 4 BC5 378 & 1150D86C & 3085EB92 & 7538ECEE & 199FB91A & 5A9614EC & 4D21FB88 & $728 F F 21 E$ \\
\hline$\underline{2} 2 \mathrm{FBFA} 2 \mathrm{E}$ & 08CE50DF & $95 \mathrm{CDE} 61 \mathrm{~F}$ & 71E5F222 & 3D30C361 & EB7676B8 & F1AE9728 & 758B70AF \\
\hline 00000000 & 00000000 & 00000000 & 00000000 & 00000000 & 00000000 & 00000000 & 00000000 \\
\hline 00000000 & 00000000 & 00000000 & 00000000 & 00000000 & 00000000 & 00000000 & 00000000 \\
\hline B4AC 9378 & 1150D86C & 3085EB92 & 7538ECEE & 199FB91A & $4 \mathrm{EC}$ & 4D21FB88 & $728 \mathrm{FF} 21 \mathrm{E}$ \\
\hline$\underline{\mathrm{A}} 2 \mathrm{FBB} \mathrm{A} 2 \mathrm{E}$ & 08CE50DF & $95 \mathrm{CDE} 61 \mathrm{~F}$ & 71E5F222 & 3D30C361 & EB7676B8 & F1AE9728 & 758B70AF \\
\hline 00000000 & 00000000 & 00000000 & 00000000 & 00000000 & 00000000 & 00000000 & 00000000 \\
\hline 00000000 & 00000000 & 00000000 & 00000000 & 00000000 & 00000000 & 00000000 & 00000000 \\
\hline
\end{tabular}




\section{B Differential Characteristic for Dynamic SHA2}

This appendix describes the differential characteristic for the third iterative part of Dynamic SHA2, used in our collision attack presented in Section 5.

A transition in Table 6 has probability $1 / 2$ when there is a difference in $a$ or $b$ and $G_{1}, G_{2}$ or $G_{3}$ is used. In this case, the difference does (not) propagate with probability $1 / 2$. When there is a difference only in $c$, it always propagates to the output of the $G$ function, independent of the function used. We also note that a difference $\Delta$ in one operand of $R$ is always transferred to $T$, and thus to $a$, except when $w_{t+1}$ or $w_{t+4}$ are $w_{8}$ or $w_{14}$, in which case the differences vanish. When two operands of $T$ have a difference $\Delta$, they cancel out and yield no difference in $T$.

The probabilities for each step assume some conditions on the message. We will take as example the first COMP when $t=2$ : we start with a difference

$$
\begin{array}{llllllll}
0 & \Delta & 0 & \Delta & 0 & 0 & 0 & 0
\end{array}
$$

in the chaining value $a, b, \ldots, h$. In the computation of COMP (first half), there is no difference in $T$, because the $\Delta$ difference in $b$ cancels that of $d$. The assignment of the new values of $f, g, h$ requires no condition on the message, for it only involves words with no difference. To obtain a difference $\Delta$ in $e$, we need that $d$ is rotated by zero bit positions, that is, we need the bits 10 to 14 of $w_{2}$ to be zero. This is easy as we have direct control over $w_{2}$. Then, to obtain no difference in $d$, we require that the difference in $b$ does not propagate in $G$. This is only possible if the Boolean function in $G$ is not $x_{1} \oplus x_{2} \oplus x_{3}$ (see Section 2.1). Since the Boolean function is determined by the last two bits of $w_{2}$, we require $w_{2}^{30} \vee w_{2}^{31}=1$, i.e., these bits should not be both zero. Now, the difference will not propagate in $G$ with probability $1 / 2$. Finally, we get a difference $\Delta$ in $c$ with probability 1.

By applying a similar reasoning to all the steps of our differential characteristic, we obtain conditions on the message $w_{0}, \ldots, w_{15}$ that are sufficient to conform to the characteristic with probability $2^{-42}$. Table 5 summarizes these conditions, along with the conditions for the other iterative parts.

Conditions on $w_{0}, \ldots, w_{7}$ ensure that in the first COMP of each step the rotations are by bit zero positions, and thus the difference remains in the MSB. The probabilities smaller than one are the probabilities that the function $G$ absorbs or passes a difference in $a$ or $b$. In the second COMP, we need some rotations to be zero in order the difference to stay in the MSB. This is achieved by setting conditions on the message, for example at $t=1$, the first ten bits of $w_{9}$ should be zero. Table 5 summarizes these conditions. After satisfying all these conditions, about 200 bits of freedom remain; indeed, besides $w_{8}$ and $w_{14}$, the message words $w_{1}$ to $w_{4}$ have to be fixed to let the symmetric $c$ and $f$ unchanged after the first iterative part.

At step $t=6$, the difference in the MSB of $w_{14}$ implies that $G$ will apply different functions to $(a, b, c)$. Similarly to Section 5.1, we will require $w_{14}^{30}=1$ and $b=$ EFFFFFFF, which will occur with probability $2^{-32}$. The MSB of $b$ should 
Table 5. Conditions on the message words $w_{0}, \ldots, w_{15}$ sufficient to follow our differential characteristic

\begin{tabular}{l|l}
\hline Word & Condition \\
\hline$w_{0}$ & - \\
\hline$w_{1}$ & $w_{1}=0$ \\
\hline$w_{2}$ & $w_{2}^{10}=\cdots=w_{2}^{14}=0, w_{2}^{25}=\cdots=w_{2}^{29}=0, w_{2}^{30} \vee w_{2}^{31}=1$ \\
\hline$w_{3}$ & $w_{3}^{30} \vee w_{3}^{31}=1$ \\
\hline$w_{4}$ & $w_{4}^{20}=\cdots=w_{4}^{29}=0, w_{4}^{30} \vee w_{4}^{31}=1$ \\
\hline$w_{5}$ & $w_{5}^{5}=\cdots=w_{5}^{9}=0$ \\
\hline$w_{6}$ & $w_{6}^{0}=\cdots=w_{6}^{4}=0, w_{6}^{15}=\cdots=w_{6}^{19}=0, w_{6}^{20}=\cdots=w_{6}^{29}=0$ \\
\hline$w_{7}$ & $w_{7}^{5}=\cdots=w_{7}^{14}=0, w_{7}^{20}=\cdots=w_{7}^{24}=0$ \\
\hline$w_{8}$ & difference in $w_{8}^{31}, w_{8}^{30}=1$ \\
\hline$w_{9}$ & $w_{9}^{0}=\cdots=w_{9}^{9}=0$ \\
\hline$w_{10}$ & $w_{10}^{5}=\cdots=w_{10}^{14}=0$ \\
\hline$w_{11}$ & $w_{11}^{15}=\cdots=w_{11}^{29}=0, w_{11}^{30} \vee w_{11}^{31}=1$ \\
\hline$w_{12}$ & $w_{12}^{10}=\cdots=w_{12}^{24}=0$ \\
\hline$w_{13}$ & $w_{13}^{0}=\cdots=w_{13}^{4}=0, w_{13}^{15}=\cdots=w_{13}^{24}=0$ \\
\hline$w_{14}$ & difference in $w_{14}^{31}, w_{14}^{10}=\cdots=w_{14}^{14}=0, w_{14}^{20}=\cdots=w_{14}^{29}=0, w_{14}^{30}=1$ \\
\hline$w_{15}$ & $w_{15}^{0}=\cdots=w_{15}^{9}=0$ \\
\hline
\end{tabular}

be zero in order the difference to propagate, which will happen with probability $1 / 2$, thus the total probability for this step $1 / 2 \times 2^{-32}=2^{-33}$.

\section{Extensions to the 512-Bit Versions}

The attacks presented in this paper can be extended to the 512-bit versions of Dynamic SHA and Dynamic SHA2 in a straightforward way. This appendix details how the attacks can be adapted.

Collision Attack on Dynamic SHA. The attack on Dynamic SHA-256 can be adapted to Dynamic SHA-512 with almost no change. Due to the different $R 1$ function, the difference word is $\Delta=8000000080000000$. Also, the probability of the local collision is lowered by about $2^{-1}$ compared to Dynamic SHA-256, as in the fifth step six rotation bits have to be fixed to zero instead of only five.

Preimage Attack on Dynamic SHA. The preimage attack on Dynamic SHA-512 is similar to that on Dynamic SHA-256, except that the 59 LSBs are determined, instead of the 28 LSBs. Then, when building the tree, $2^{224}$ solutions are expected, 
Table 6. Differential characteristic for the third iterative part of Dynamic SHA2. The difference at step $t$ is the difference in the state before computing step $t$. The column $T$ indicates the difference in the temporary variable $T$. The probability on a line is the probability to reach the next difference, when conditions on the message are satisfied.

\begin{tabular}{|c|c|c|c|c|c|c|c|c|c|c|c|}
\hline$t$ & (message input) & $a$ & $b$ & $c$ & $d$ & $e$ & $f$ & $g$ & $h$ & $T$ & prob. \\
\hline \multirow{2}{*}{1} & & 0 & 0 & 0 & $\Delta$ & 0 & 0 & $\Delta$ & 0 & 0 & 1 \\
\hline & $\left(w_{1}, \ldots, w_{0}\right)$ & 0 & 0 & 0 & 0 & $\Delta$ & 0 & 0 & $\Delta$ & 0 & 1 \\
\hline \multirow{2}{*}{1} & & 0 & 0 & 0 & 0 & 0 & $\Delta$ & 0 & 0 & $\Delta$ & 1 \\
\hline & $\left(w_{9}, \ldots\right.$ & $\Delta$ & 0 & 0 & 0 & 0 & 0 & $\Delta$ & 0 & 0 & $2^{-1}$ \\
\hline \multirow{2}{*}{2} & $\left(\begin{array}{lll}\mu_{1} & \mu_{1}\end{array}\right)$ & 0 & $\Delta$ & 0 & $\Delta$ & 0 & 0 & 0 & 0 & 0 & $2^{-1}$ \\
\hline & & 0 & 0 & $\Delta$ & 0 & $\Delta$ & 0 & 0 & 0 & 0 & 1 \\
\hline \multirow{2}{*}{2} & & 0 & 0 & 0 & $\Delta$ & 0 & $\Delta$ & 0 & 0 & 0 & 1 \\
\hline & $\left(w_{10}, \ldots, w_{9}\right)$ & 0 & 0 & 0 & 0 & $\Delta$ & 0 & $\Delta$ & 0 & 0 & 1 \\
\hline \multirow{2}{*}{3} & & $\Delta$ & 0 & 0 & 0 & 0 & 0 & 0 & $\Delta$ & 0 & $2^{-1}$ \\
\hline & $\left(w_{3}, \ldots, w_{2}\right)$ & 0 & $\Delta$ & 0 & 0 & 0 & 0 & 0 & 0 & $\Delta$ & $2^{-1}$ \\
\hline \multirow{2}{*}{3} & (ט) & $\Delta$ & 0 & $\Delta$ & 0 & 0 & 0 & 0 & 0 & 0 & $2^{-1}$ \\
\hline & $\left.\cdot, w_{10}\right)$ & 0 & $\Delta$ & 0 & $\Delta$ & 0 & $\Delta$ & 0 & 0 & $\Delta$ & $2^{-1}$ \\
\hline \multirow{2}{*}{4} & & $\Delta$ & 0 & $\Delta$ & 0 & $\Delta$ & 0 & $\Delta$ & 0 & 0 & $2^{-1}$ \\
\hline & $\left(w_{4}, \ldots, w_{3}\right)$ & 0 & $\Delta$ & 0 & $\Delta$ & 0 & $\Delta$ & 0 & $\Delta$ & 0 & 1 \\
\hline \multirow{2}{*}{4} & $\left.\mu_{1}\right)_{1}$ & 0 & 0 & $\Delta$ & $\Delta$ & $\Delta$ & 0 & $\Delta$ & 0 & 0 & 1 \\
\hline & $\left(w_{12}\right.$ & 0 & 0 & 0 & 0 & $\Delta$ & $\Delta$ & 0 & $\Delta$ & $\Delta$ & 1 \\
\hline \multirow{2}{*}{5} & $\left.w_{1}\right)$ & 0 & 0 & 0 & 0 & 0 & $\Delta$ & $\Delta$ & 0 & 0 & 1 \\
\hline & & 0 & 0 & 0 & 0 & 0 & 0 & $\Delta$ & $\Delta$ & 0 & 1 \\
\hline \multirow{2}{*}{5} & $\left(w_{13}, \ldots, w_{12}\right.$ & 0 & 0 & 0 & 0 & 0 & 0 & 0 & $\Delta$ & $\Delta$ & 1 \\
\hline & $\left(w_{13}, \ldots, w_{12}\right.$ & 0 & 0 & 0 & 0 & 0 & $\Delta$ & 0 & 0 & $\Delta$ & 1 \\
\hline \multirow{2}{*}{6} & $\left(u_{6} \quad u_{5}\right)$ & $\Delta$ & 0 & 0 & 0 & 0 & 0 & $\Delta$ & 0 & 0 & 1 \\
\hline & & 0 & $\Delta$ & 0 & $\Delta$ & 0 & 0 & 0 & $\Delta$ & $\Delta$ & $2^{-1}$ \\
\hline \multirow{2}{*}{6} & $\left(w_{14}, \ldots w_{13}\right)$ & $\Delta$ & 0 & $\Delta$ & $\Delta$ & $\Delta$ & 0 & 0 & 0 & $\Delta$ & $2^{-33}$ \\
\hline & & 0 & $\Delta$ & 0 & $\Delta$ & $\Delta$ & $\Delta$ & 0 & 0 & 0 & $2^{-1}$ \\
\hline \multirow{2}{*}{7} & $\left(w_{7}, w_{6}\right)$ & 0 & 0 & 0 & $\Delta$ & $\Delta$ & $\Delta$ & $\Delta$ & 0 & 0 & 1 \\
\hline & $\left(w_{7}, \ldots, w_{6}\right)$ & 0 & 0 & 0 & 0 & $\Delta$ & $\Delta$ & $\Delta$ & $\Delta$ & 0 & 1 \\
\hline \multirow{3}{*}{7} & ( & 0 & 0 & 0 & 0 & 0 & $\Delta$ & $\Delta$ & $\Delta$ & $\Delta$ & 1 \\
\hline & $\left(w_{15}, \ldots, w_{14}\right.$ & 0 & 0 & 0 & 0 & 0 & 0 & $\Delta$ & $\Delta$ & 0 & 1 \\
\hline & & 0 & 0 & 0 & 0 & 0 & 0 & 0 & 0 & & \\
\hline
\end{tabular}

leading to an attack complexity of $2^{256}$ on the compression function. Calculations for preimages on the full hash function (with correct padding bits) give a cost of of $2^{262}$ compression function evaluations.

Collision Attack on Dynamic SHA2. To attack Dynamic SHA2-512 we use a similar differential path. The changes are that the condition on the first block is on 64 bits (starting from a chaining value with $b=$ FFFFFFFFFFFFFFFF), the fact that in the second iterative part the probability is $2^{-6}$ for each of the 
Table 7. Conditions on the message words $w_{0}, \ldots, w_{15}$ sufficient to follow our differential characteristic in Dynamic SHA2-512

\begin{tabular}{l|l}
\hline Word & Condition \\
\hline$w_{0}$ & - \\
\hline$w_{1}$ & $w_{1}=0, w_{1}^{18}=\cdots=w_{1}^{23}=0, w_{1}^{42}=\cdots=w_{1}^{47}=0, w_{1}^{60}=1, w_{1}^{61}=0$ \\
\hline$w_{2}$ & $w_{2}^{18}=\cdots=w_{2}^{29}=0, w_{2}^{42}=\cdots=w_{2}^{47}=0, w_{2}^{60}=0, w_{2}^{61}=1, w_{2}^{62} \vee w_{2}^{63}=1$ \\
\hline$w_{3}$ & $w_{3}^{54}=\cdots w_{3}^{59}=0, w_{3}^{60}=w_{3}^{61}=1, w_{3}^{62} \vee w_{3}^{63}=1$ \\
\hline$w_{4}$ & $w_{4}^{6}=\cdots=w_{4}^{11}=0, w_{4}^{18}=\cdots=w_{4}^{23}=0, w_{4}^{42}=\cdots=w_{4}^{47}=0$, \\
\hline$w_{5}$ & $w_{5}^{60}=\cdots=w_{4}^{61}=0, w_{4}^{62} \vee w_{4}^{63}=1$ \\
\hline$w_{6}$ & $w_{6}^{48}=\cdots=w_{5}^{53}=0, w_{5}^{60}=1, w_{5}^{61}=1$ \\
\hline$w_{7}$ & $w_{7}^{6}=\cdots=w_{7}^{23}=0, w_{7}^{36}=\cdots=w_{6}^{60}=\cdots$ \\
\hline$w_{8}$ & difference in $w_{8}^{63}, w_{8}^{62}=1$ \\
\hline$w_{9}$ & $w_{9}^{12}=\cdots=w_{9}^{17}=0, w_{9}^{36}=\cdot=w_{9}^{41}=0, w_{9}^{60}=1, w_{9}^{61}=0$ \\
\hline$w_{10}$ & $w_{10}^{6}=\cdots=w_{10}^{11}=0, w_{10}^{18}=\cdots=w_{10}^{23}=0, w_{10}^{42}=\cdots=w_{1}^{61}=1$ \\
\hline$w_{11}$ & $w_{10}^{61}=1$ \\
\hline$w_{12}$ & $w_{11}^{12}=\cdots=\cdots=w_{11}^{41}=0, w_{11}^{48}=\cdots=w_{11}^{63}=\cdots=0, w_{11}^{60}=w_{11}^{60}=0, w_{12}^{36}=\cdots=w_{12}^{67}=0, w_{12}^{60}=w_{12}^{61}=0$ \\
\hline$w_{13}$ & $w_{13}^{36}=\cdots=w_{13}^{41}=0, w_{13}^{60}=1, w_{13}^{61}=0$ \\
\hline$w_{14}$ & difference in $w_{14}^{63}, w_{14}^{12}=\cdots=w_{11}^{63}=1$ \\
\hline$w_{15}$ & $w_{15}^{6}=\cdots=w_{15}^{11}=0, w_{15}^{36}=\cdots=w_{15}^{41}=0, w_{15}^{60}=w_{15}^{61}=1$ \\
\hline
\end{tabular}

two transitions, the decrease in the probability only of the sixth COMP from $2^{-33}$ to $2^{-65}$, and the different set of conditions on the message described in Table 7. Hence, the total time complexity of this attack is $2^{85}$. We note that in this approach the attack fixes $w_{i}^{60}$ and $w_{i}^{61}$ to $i \bmod 4$ (which causes the same function to be used in this case as in the attack on Dynamic SHA2-256). 